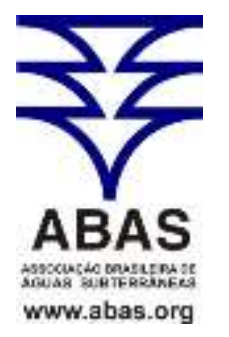

\title{
ESTIMATIVA DA VELOCIDADE DE FLUXO SUBTERRÂNEO POR IMAGEAMENTO ELÉTRICO
}

\section{UNDERGROUND FLOW VELOCITY ESTIMATE BY ELECTRI- CAL IMAGING}

Artigo recebido em: 20/11/2014 e aceito para publicação em: 12/02/2015.

DOI: http://dx.doi.org/10.14295/ras.v29i1.27990

\begin{abstract}
This paper describes a field procedure to estimate the groundwater flow velocity in the vadose zone using the electrical imaging geophysical methodology. During the procedure, subsurface resistivity images were obtained for different times by surface measurements of apparent resistivity along a profile. The underground flow was traced by observing the change in subsurface resistivity caused by the movement of saline water injected in two shallow holes. The estimated velocity for the flow of injected saline water was compared to the velocity obtained through conventional assays of permeability (drawdown tests) conducted in the study area and can be considered satisfactory. The procedure is important because it can be used for monitoring environmental contamination, and does not require the drilling of wells.
\end{abstract}

Keywords: Electrical Resistivity Imaging. Groundwater Flow. Hydraulic Conductivity. Time-lapse Electrical Resistivity.

Resumo: Este artigo descreve um procedimento de campo para estimar a velocidade do fluxo de água subterrânea na zona vadosa usando a metodologia geofísica do imageamento elétrico. Durante a aplicação do procedimento, imagens da distribuição de resistividade em subsuperfície foram obtidas para diferentes tempos por meio de medições de resistividade aparente realizadas na superfície do terreno ao longo de um perfil. O fluxo subterrâneo foi rastreado pela observação da variação na resistividade da subsuperfície causada pelo movimento de água salina injetada em dois poços rasos. A velocidade estimada para o fluxo da água salina injetada foi comparada com a velocidade obtida por meio de ensaios convencionais de permeabilidade (ensaios de rebaixamento) realizados na área do estudo e pode ser considerada como satisfatória. O procedimento é importante porque pode ser usado no monitoramento de contaminação do meio ambiente, além de não necessitar da perfuração de poços tubulares.

Palavras-chave: Imageamento Elétrico. Fluxo de Água Subterrânea. Condutividade Hidráulica.Variação da Resistividade Elétrica com o Tempo.

\section{INTRODUÇÃO}

A Geofísica tem um papel importante nos estudos desenvolvidos para água subterrânea. A principal aplicação da metodologia geofísica na prospecção de água subterrânea é voltada para a procura de rochas, estruturas ou ambientes geológicos que possam permitir a extração de água (LIMA, 1990; MEDEIROS e LIMA, 1990; HARARI, 1996; SOUZA FILHO et al., 2006; LIMA, 2010; OLIVEIRA, 2011; SOUSA e LUIZ, 2012; MENDES et al., 2014). Os métodos geofísicos podem, porém, ainda ser usados para: a) Estimar características físicas dos aquíferos, como porosidade, condutividade hidráulica, per- meabilidade e transmissividade (GRIFFITHS, 1976; KELLY, 1977; PONZINI et al., 1984; LIMA e NIWAS, 2000; NIWAS e LIMA, 2003; LU e SATO, 2007; SOUPIOS et al., 2007); (b) Indicar alguns parâmetros de potabilidade da água, como, por exemplo, seu grau de salinidade (HAGREY e MÜLLER, 2000; BENKABBOUR et al., 2004) ou contaminações por poluentes químicos e orgânicos (COSTA et al., 1995; BENSON et al., 1997; SAUCK et al., 1998; NUNES e LUIZ, 2006; BAESSA et al., 2010; BAHIA et al., 2011; CUNHA e SHIRAIWA, 2011).

\footnotetext{
${ }^{1}$ Eton Institute, Dubai, Emirados Árabes (anapaulavb.neves@ hotmail.com)

${ }^{2}$ Universidade Federal do Pará (gouvea@ufpa.br)
} 
Mais recentemente, a Geofísica, através do Método da Eletrorresistividade, tem sido aplicada para monitorar o comportamento da subsuperfície. Nesse tipo de aplicação, são realizadas medidas periódicas de resistividade na superfície do terreno ou em poços. Trabalhos envolvendo essa aplicação foram desenvolvidos por French e Binley (2004), para monitorar a infiltração de água proveniente do derretimento de neve; Cassiani et al. (2006), para monitorar possível conexão hidráulica entre um aquífero raso não confinado e um aquífero confinado profundo; De Franco et al. (2009), para monitorar intrusão salina na costa da Lagoa de Veneza, na Itália; Agramakova (2011), para verificar a estabilidade estrutural do topo de uma caverna e para avaliar o potencial das medidas na exploração de água subterrânea; e Farooq et al. (2014), para monitorar a injeção de cimento visando tamponar cavidades no subsolo.

No presente trabalho, aplicou-se essa metodologia em medidas na superfície do terreno com o objetivo de estimar a velocidade de fluxo na zona não saturada da subsuperfície. $\mathrm{O}$ procedimento pode simplificar a obtenção da estimativa da velocidade do fluxo por não ser necessária a determinação dos parâmetros gradiente hidráulico, condutividade hidráulica e porosidade do aquífero, que normalmente requerem um procedimento que demanda tempo e labor, pois requer a coleta de amostras e observações em poços tubulares (FEITOSA et al., 2008; FETTER, 2001).

Durante o estudo, foram realizadas medidas de resistividade ao longo de um perfil e obtidas imagens geoelétricas da distribuição de resistividade na subsuperfície. O controle do fluxo subterrâneo foi efetuado amostrando-se periodicamente o perfil após a injeção de água salinizada em dois pontos. A água salinizada permitiu que se visualizasse o fluxo subterrâneo por observação de variações na resistividade elétrica da subsuperfície causada pela passagem da água salinizada injetada. Procedimento semelhante foi realizado por Barker e Moore (1998) na Inglaterra envolvendo a injeção de água no solo e acompanhamento da sua migração em subsuperfície por meio de medidas de resistividade realizadas em diferentes tempos. No mesmo trabalho, esses autores também realizaram medidas de resistividade durante o bombeamento de um poço. Entretanto, durante os experimentos, Barker e Moore (1998) apenas observaram o fluxo subterrâneo sem calcular a velocidade de movimentação da água, como foi realizado no presente estudo. Por outro lado, Wilkinson et al. (2010) e Doetsch et al. (2012) desenvolveram trabalhos em que a velocidade de fluxo é estimada a partir das medidas temporais de resistividade. Os primeiros autores realizaram seus experimentos com eletrodos colocados em um poço e analisaram o fluxo em aquífero confinado; já os outros autores realizaram medidas na superfície do terreno, monitorando solução salina injetada na zona saturada de um aquífero.

A velocidade do fluxo subterrâneo é um parâmetro importante para a hidrogeologia de uma região e vem tornando-se muito importante nos estudos de contaminação do meio ambiente, pois pode fornecer dados sobre a velocidade de migração de contaminantes na subsuperfície.

O presente estudo foi desenvolvido na Área de Depósito de Rejeitos Sólidos (ADRS) da ALBRAS (Alumínio Brasileiro S.A.), localizada no município de Barcarena, estado do Pará. Os ADRS são locais onde são depositados os rejeitos sólidos provenientes do processamento de alumina para obtenção de alumínio industrial. Esses rejeitos são ricos em compostos químicos (principalmente sulfato e fluoreto) que, quando passam para o solo, podem poluir a água subterrânea dos aquíferos rasos e chegar ao homem causando-lhe problemas de saúde.

\section{LOCALIZAÇÃO DA ÁREA DE ESTUDO}

A ADRS da ALBRAS localiza-se no município de Barcarena, estado do Pará, $30 \mathrm{~km}$ a sudoeste de Belém, a capital do estado. A área está posicionada nas coordenadas $1^{\circ} 40^{\prime} 18.04 " \mathrm{~S}$, $48^{\circ} 37^{\prime} 40.12^{\prime \prime} \mathrm{O}$, no cruzamento das rodovias PA481 e PA-483 (Figura 1). 

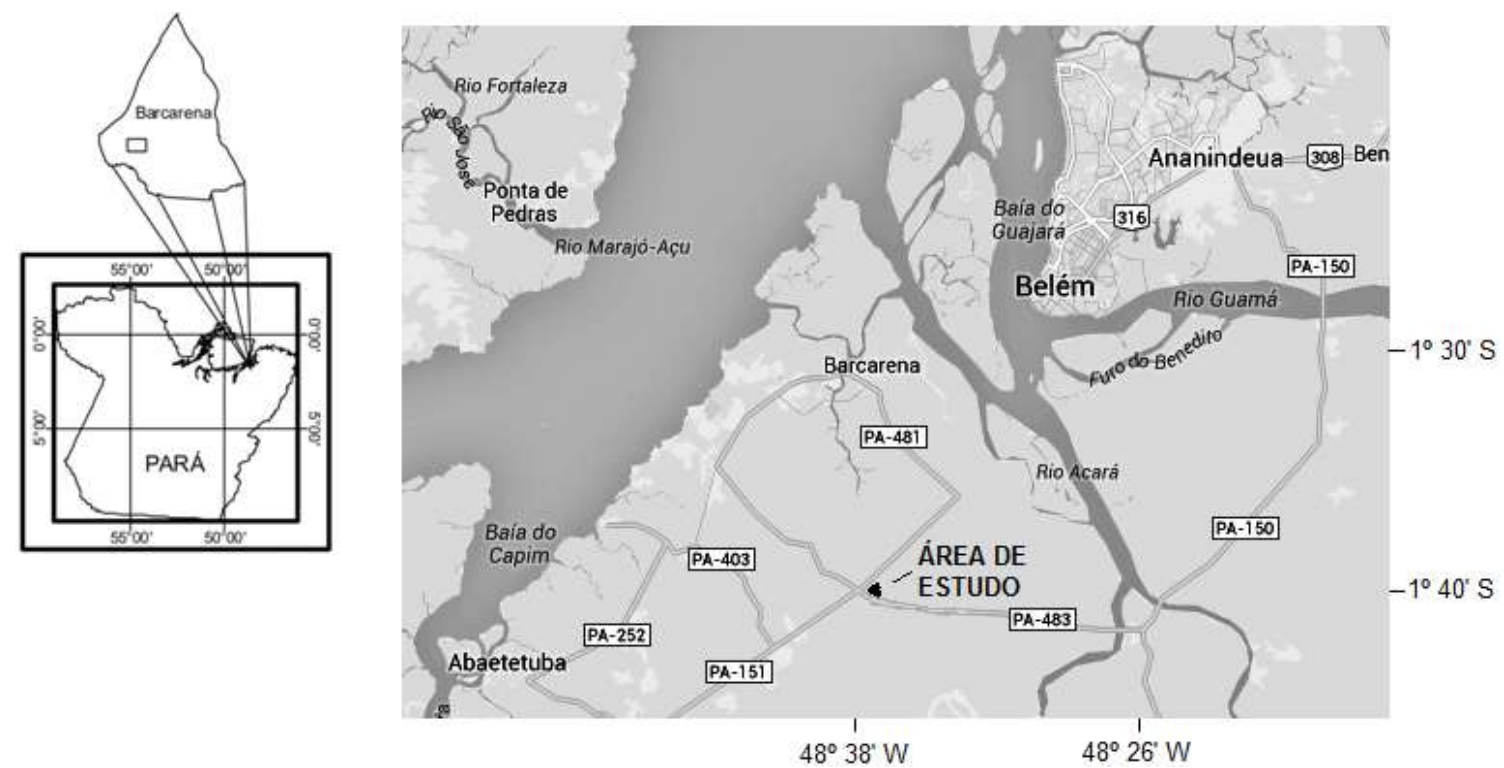

Figura 1 - Mapa de localização

Figure 1 - Location map

\subsection{Geologia e Hidrogeologia da Área}

De acordo com Rodrigues (1999), a geologia no local na área de estudo é representada por sedimentos aluvionares quaternários (sedimentos Pós-Barreiras) sobrepostos a sedimentos fluviolacustrinos terciários da Formação Barreiras. Estudos realizados em poços indicam que os sedimentos aluvionares quaternários ocorrem até profundidades de aproximadamente 12 metros e sos, argilo-arenosos, silte-areno-argilosos, de coloração esbranquiçada, amarelada e avermelhada. Observam-se, ainda, em torno dos $6 \mathrm{~m}$ de profundidade, níveis de concreções lateríticas e, à cerca dos $8 \mathrm{~m}$ de profundidade, seixos concrecionários (RODRIGUES, 1999). A Figura 2 mostra o perfil litológico de um poço localizado na área de estudo.

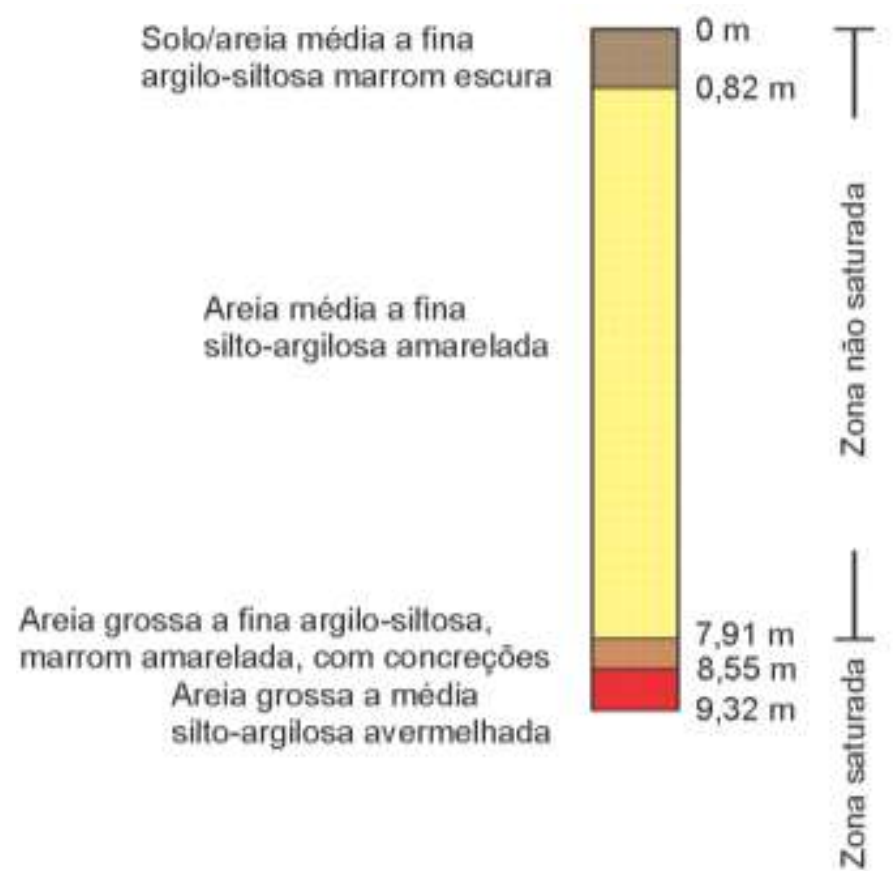

Figura 2 - Perfil litológico de poço da área de estudo (adaptado de Rodrigues, 1999) Figure 2 - Lithological profile of well in the study area (adapted from Roberts, 1999) 
Estudos realizados pelo Instituto de Pesquisas Tecnológicas do Estado de São Paulo (IPT, 1984), concluíram que na região de Barcarena existem dois sistemas aquíferos: um, superior (livre com cobertura), com matriz arenosa fina a média e de espessura variável; e outro, inferior (semiconfinado), com matriz arenosa grosseira e heterogênea, apresentando espessura relativamente constante. Os estudos indicaram que o bombeamento do aquífero inferior pode ocasionar uma infiltração vertical descendente das águas do aquífero superior através da camada semiconfinante que, por sua vez, tende a restabelecer os volumes bombeados. Em tal situação, tanto o aquífero inferior, como o superior, estariam sujeitos ao aporte de água por drenança vertical descendente (IPT, 1984).

Segundo Rodrigues (1999), os aquíferos livres da região de Barcarena são constituídos pelos sedimentos aluvionares do quaternário (Pós-Barreiras) e pelos sedimentos terciários da Formação Barreiras. Os primeiros são compostos por areias, areias argilosas, argilas arenosas e siltes, ocorrendo até 10 metros de profundidade. $\mathrm{Na}$ área do presente estudo, o topo do aquífero livre é encontrado entre 5,5 e $9 \mathrm{~m}$ de profundidade. Ensaios de permeabilidade realizados através de testes de rebaixamento na zona não saturada de três poços rasos na área de estudo mostraram os seguintes valores de condutividade hidráulica: $1,44 \times 10^{-4} \mathrm{~cm} / \mathrm{s}, 1,54 \times 10^{-4} \mathrm{~cm} / \mathrm{s}$ e $2,52 \times 10^{-4}$ $\mathrm{cm} / \mathrm{s}$ (Rodrigues, 1999).

Os aquíferos mais superficiais da Formação Barreiras possuem espessuras máximas de 15 $\mathrm{m}$ e pequena continuidade lateral, estando localmente cobertos por camadas de argila, silte, argila arenosa e areia argilosa, (IPT, 1984; TANCREDI, 1996). As vazões dos aquíferos do Barreiras variam de $140 \mathrm{~m}^{3} / \mathrm{h}$ a $290 \mathrm{~m}^{3} / \mathrm{h}$ (IPT, 1984).

As águas subterrâneas de Barcarena apresentam acidez relativamente alta $(\mathrm{pH}$ geralmente inferior a 5), baixa dureza (geralmente abaixo de $2 \mathrm{ppm}$ ), baixa condutividade elétrica (abaixo de $45 \square \mathrm{S} / \mathrm{cm}$ ), pobreza de determinados cátions (especialmente de cálcio) e teores de ferro relativamente elevados (maiores que $0,3 \mathrm{mg} / \mathrm{l}$ ) em alguns pontos, porém, podem ser consideradas de potabilidade aceitável para o consumo humano. Essas águas são classificadas como águas doces, brandas, ácidas, do tipo bicarbonatadas sódicas (LIMA e KOBAYASHI, 1988).

\subsection{Método da Eletrorresistividade}

A operação com o método da eletrorre- sistividade consiste em injetar corrente contínua (ou de baixa frequência) por meio de contato galvânico em dois pontos do terreno (denominados de A e B ou eletrodos de corrente) e medir a diferença de potencial entre dois outros eletrodos, também por meio de contato galvânico (denominados $\mathrm{M}$ e $\mathrm{N}$ ou eletrodos de potencial). $\mathrm{O}$ conhecimento da corrente injetada (I), da diferença de potencial $(\Delta \mathrm{V})$ e das posições relativas dos pontos A, B, M e N permite que se calcule o valor da resistividade $(\rho)$ dos materiais que se encontram abaixo da superfície dos terrenos. Esse valor, para um meio homogêneo e isotrópico, é dado por:

$$
\rho=\frac{\Delta V}{I} K,
$$

sendo

$$
K=\frac{6,28}{\frac{1}{A M}-\frac{1}{B M} \frac{1}{A N}+\frac{1}{B N}}
$$

o fator geométrico.

Como o ambiente geológico não é homogêneo, ele apresenta uma distribuição de resistividade que pode variar vertical e lateralmente. Por isso, os valores de resistividade obtidos durante a realização de medidas não representam os valores das resistividades verdadeiras das camadas geológicas da subsuperfície, muito embora estejam relacionados a eles. O que se obtém são valores denominados de resistividade aparente.

Dois procedimentos são comumente empregados nas medidas de resistividade aparente: Sondagem Elétrica Vertical (SEV) e Caminhamento Elétrico (Orellana, 1982). Na SEV, os eletrodos de corrente geralmente são deslocados simetricamente em relação a um ponto central. Este procedimento permite que se conheça a distribuição da resistividade com a profundidade. No Caminhamento Elétrico, normalmente, todos os eletrodos são deslocados lateralmente mantendo-se constante a distância entre eles. Este procedimento mostra a variação lateral da resistividade a uma determinada profundidade.

Os eletrodos de injeção de corrente e de medida da diferença de potencial podem ser colocados em diversas posições padronizadas denominadas de arranjos de eletrodos. No presente trabalho os eletrodos foram colocados segundo o arranjo dipolo-dipolo, em que os eletrodos de corrente são dispostos adjacentes aos eletrodos de potencial. Na Figura 3 são mostrados três posicionamentos de eletrodos para o arranjo dipolodipolo. $\mathrm{O}$ posicionamento mostrado na parte su- 
perior da figura permite que se investigue a menor profundidade da subsuperfície, enquanto o posicionamento da parte inferior da figura permite a investigação da maior profundidade. Durante as medidas realizadas neste trabalho, foram em- pregados 5 posicionamentos, cada um investigando uma determinada profundidade, o que permitiu obter a variação lateral da resistividade em 5 níveis de profundidade e a construção de uma imagem da distribuição de resistividade.

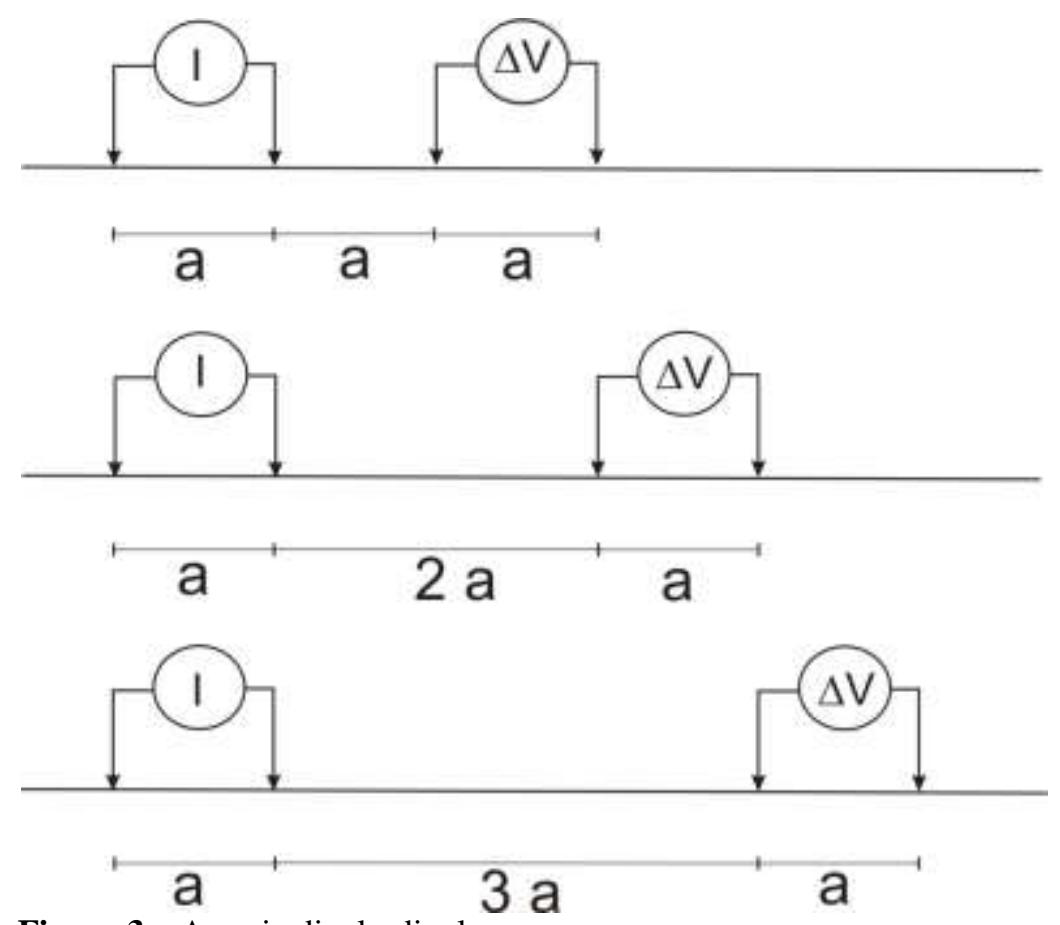

Figura 3 - Arranjo dipolo-dipolo

Figure 3 - Dipole-dipole array

\section{MATERIAIS E MÉTODOS}

Para a realização deste estudo foi montado um experimento em que foram infiltrados no subsolo 360 litros de solução salina contendo 56 g/l de cloreto de sódio. A opção por solução salina se deu para facilitar a observação da variação de resistividade, que indica o movimento da água. $\mathrm{O}$ experimento durou 15 dias.

Para o experimento de infiltração foi estabelecida uma linha de levantamento de direção $\mathrm{N} 20^{\circ}$ onde foram colocados vinte eletrodos espaçados de $2 \mathrm{~m}$. A injeção da solução salina foi feita, por gravidade, a partir de dois tambores com capacidade de 180 litros, em dois poços ra- sos, com diâmetro de $25 \mathrm{~cm}$, na linha de levantamento geofísico. O primeiro poço encontravase na posição $9 \mathrm{~m}$ e tinha $70 \mathrm{~cm}$ de profundidade; o segundo encontrava-se na posição $23 \mathrm{~m}$ e possuía $50 \mathrm{~cm}$ de profundidade. Em cada poço foram injetados 180 litros da solução. O tempo de injeção foi 607 minutos. A Figura 4 mostra um dos poços de injeção e a posição de dois eletrodos, enquanto na Figura 5 é mostrado um dos tambores de 180 litros, a partir do qual a solução salina foi drenada para o subsolo. A base dos tambores foi colocada $90 \mathrm{~cm}$ acima do solo, sobre uma mesa. 


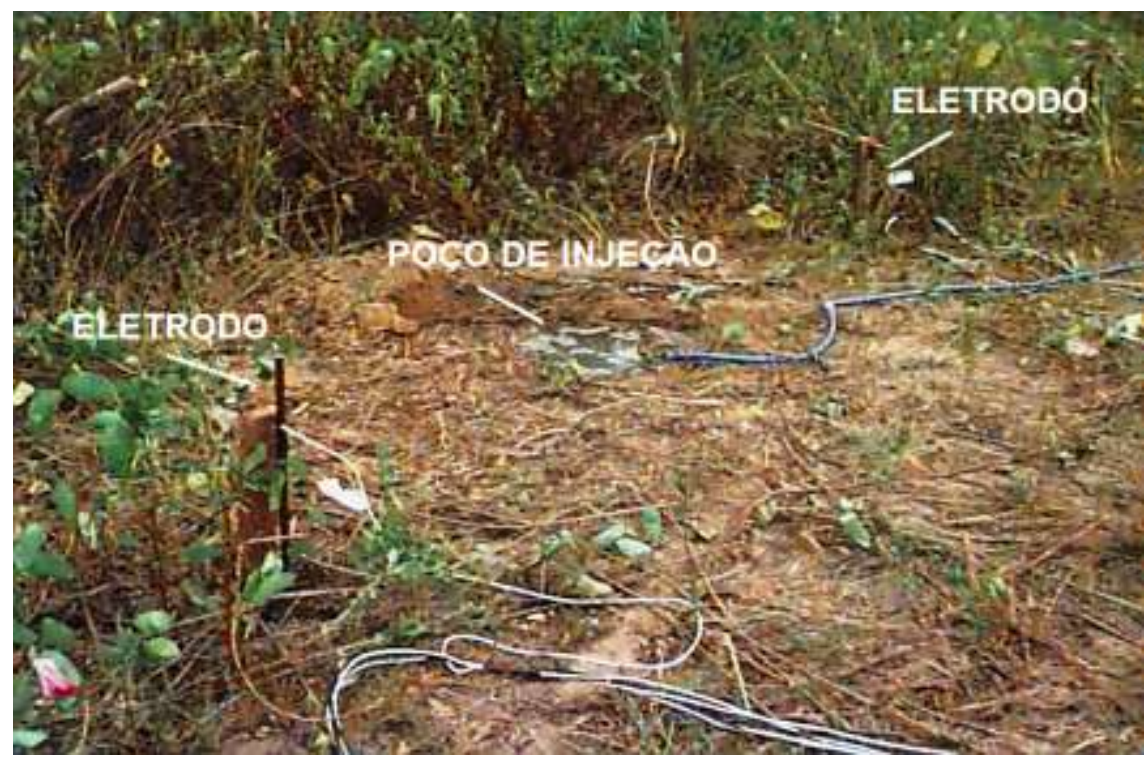

Figura 4 - Poço raso utilizado para a injeção da solução salina

Figure 4 - Shallow hole used for injection of the saline solution

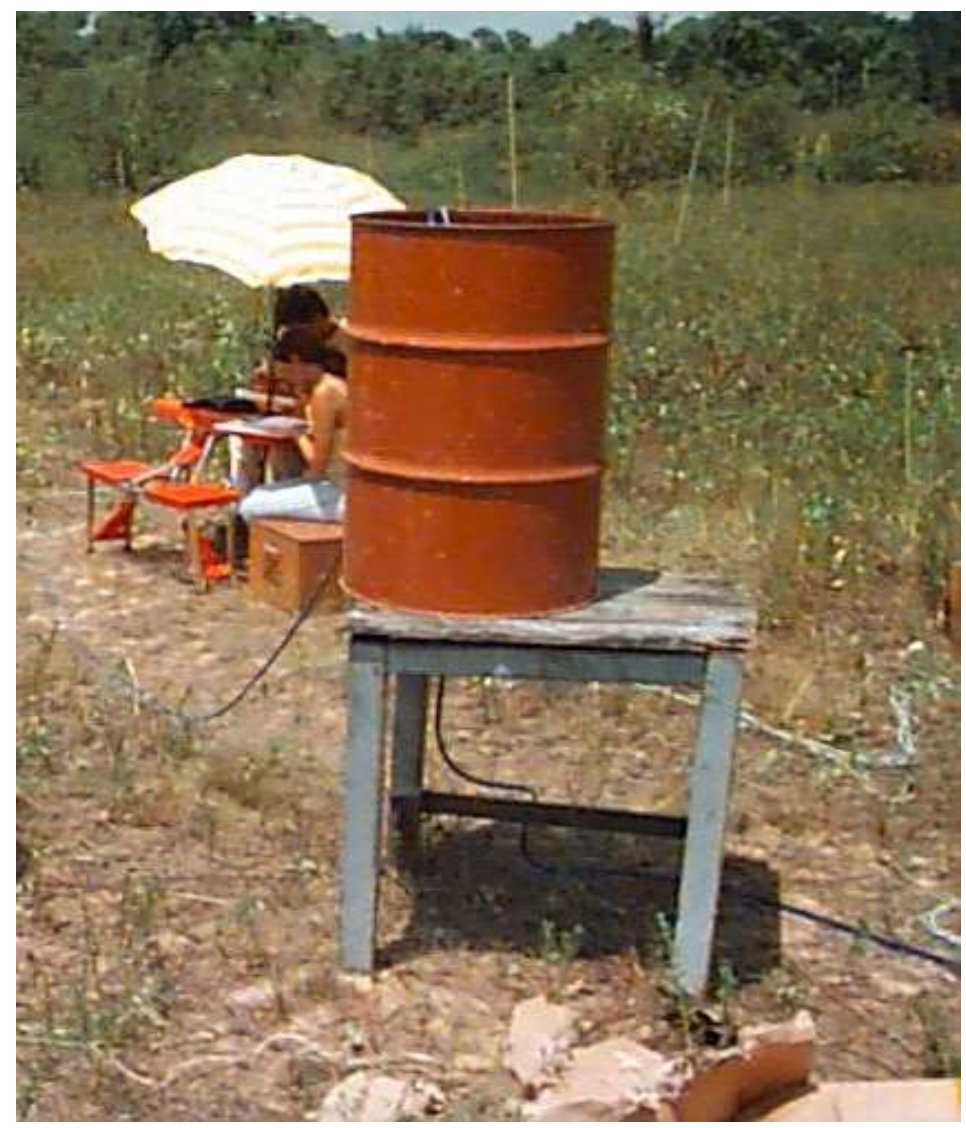

Figura 5 - Tambor de 180 litros usado para drenar a solução salina no subsolo Figure 5 - Drum of 180 liters used to drain the saline solution underground

Os vinte eletrodos colocados na linha de levantamento foram utilizados para a realização de medidas de resistividade, com o objetivo de acompanhar as variações provocadas pela injeção da solução salina e, assim, monitorar a sua distri- buição na subsuperfície.

As medidas de resistividade foram realizadas com o procedimento de Caminhamento Elétrico usando o arranjo dipolo-dipolo com dipolos de $2 \mathrm{~m}$ e cinco níveis de investigação. A 
operação de campo com este arranjo permitiu que se investigasse a variação vertical e lateral da resistividade, gerando imagens da distribuição de resistividade na subsuperfície (imageamento elétrico). $\mathrm{O}$ equipamento utilizado foi o resisti- vímetro GEOTEST, que possui um único canal. Com o objetivo de facilitar a operação de coleta de dados, uma unidade de chaveamento manual foi acoplada ao resistivímetro e conectada aos vinte eletrodos (Figura 6).

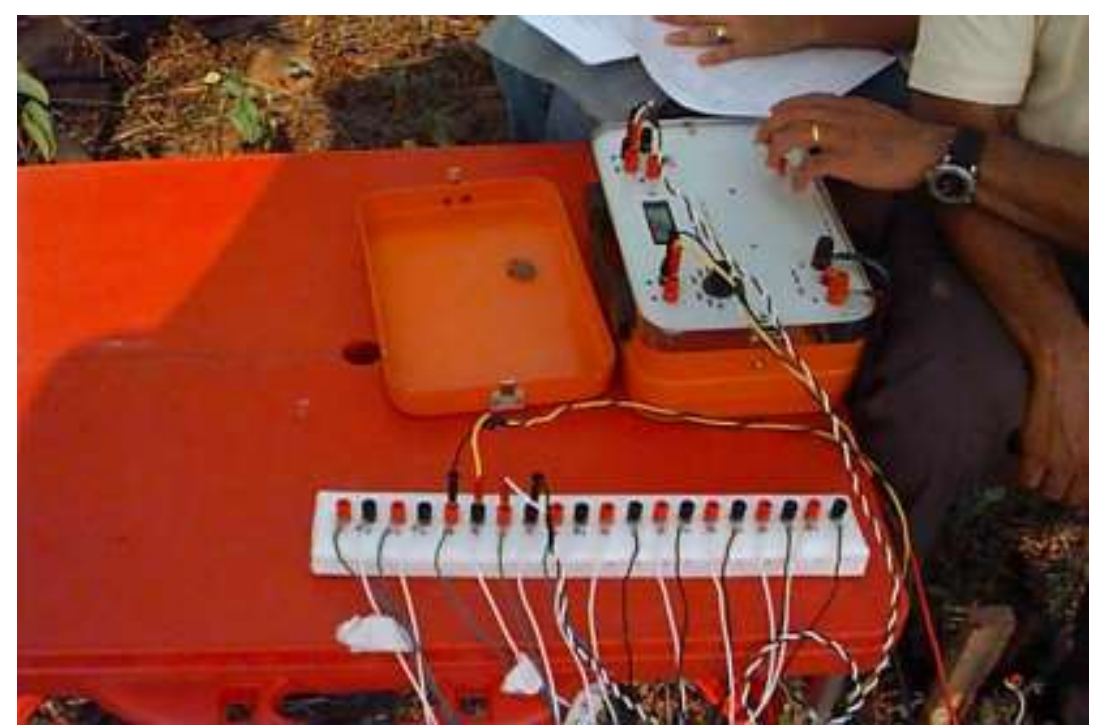

Figura 6 - Equipamento e unidade de chaveamento usados nas medidas de resistividade Figure 6 - Equipment and switching unity used in the resistivity measurements

As medidas de resistividade aparente obtidas no imageamento elétrico foram interpretadas através da técnica de inversão automática a fim de obterem-se modelos interpretativos bidimensionais, que mostrassem a distribuição de resistividade na subsuperfície. O processo de inversão foi realizado com o programa RES2DINV, versão 3.4, da Geotomo Software, que utiliza o método dos mínimos quadrados. Durante o processo de inversão é estabelecido um modelo de distribuição de resistividade para a subsuperfíce e calculada a resposta do modelo em termos de resistividade aparente. A seguir, a resposta do modelo é comparada com os valores de resistividade aparente medidos; caso a diferença entre os valores medidos e os calculados seja grande (rms - erro médio quadrático elevado), novo modelo é gerado iterativamente e calculada a sua resposta. O objetivo é chegar a um modelo que produza um erro pequeno ou que não mais varie. Neste trabalho, o rms máximo obtido no processo de inversão foi de $3,8 \%$.

Antes de iniciar a injeção, a linha de levantamento foi amostrada três vezes para obter- se a distribuição do "background" dos valores de resistividade ao longo do perfil. As medidas de monitoramento da injeção foram realizadas: (a) durante a infiltração a intervalos de 2 horas; e (b) após a injeção dos 360 litros de solução, em dias alternados, sendo realizados, a cada dia, dois perfis de medidas espaçados de 2 horas. Em cada amostragem do perfil foram executadas 75 medidas de resistividade aparente, realizadas em cerca de 30 minutos.

\section{RESULTADOS}

A distribuição normal do "background" da resistividade ao longo da linha de levantamento, antes de iniciar-se a injeção de solução salina, foi obtida a partir da média aritmética de três amostragens. $\mathrm{O}$ modelo de subsuperfície antes da injeção é ilustrado na Figura 7. Esse modelo é a referência para observação dos efeitos causados na distribuição de resistividade pela injeção da solução salina. 


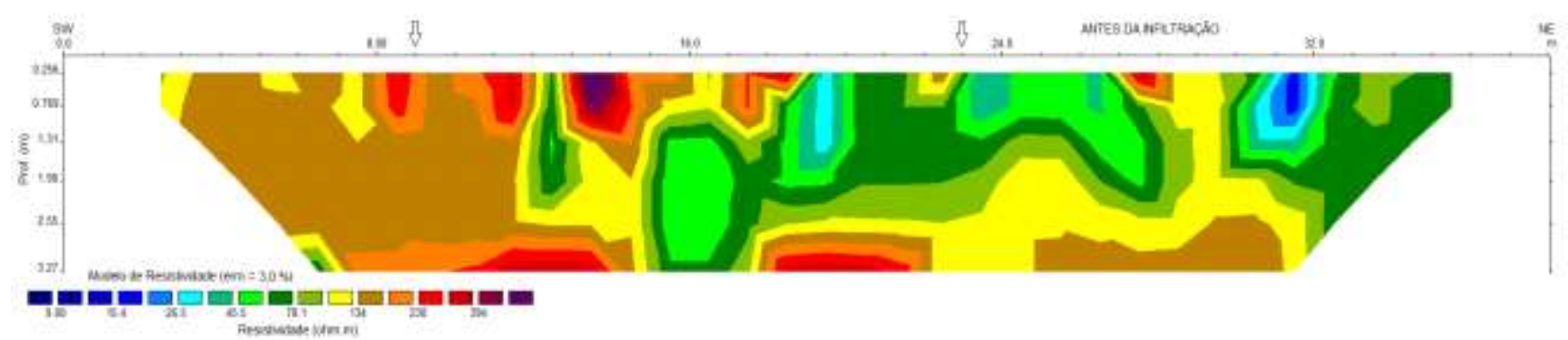

Figura 7 - Modelo de resistividade para a subsuperfície obtido a partir da média de três amostragens, realizadas antes de iniciar a injeção

Figure 7 - Electrical subsurface model obtained from the average of three samples, performed before starting the injection

Uma hora após iniciada a injeção, foi realizado o primeiro perfil de medidas, cujo modelo está representado na Figura 8. Observa-se que ocorre uma diminuição dos valores da resistividade entre as posições 8 e $10 \mathrm{~m}$ e entre as posições 22 e $24 \mathrm{~m}$, em relação à imagem do modelo antes da injeção (Figura 7), diretamente abaixo dos pontos de infiltração da solução salina $(9 \mathrm{~m}$ e $23 \mathrm{~m}$ ). Com o passar do tempo (Figuras 9 a 12), observa-se um espalhamento desses valores mais baixos, indicando que esses são os sentidos do fluxo subterrâneo nos locais de injeção. O espalhamento dos valores mais baixos de resistividade é mais visível entre as posições 22 e $24 \mathrm{~m}$ do que entre as posições 8 e $10 \mathrm{~m}$, o que sugere uma maior permeabilidade do material depositado nas proximidades das posições 22 e $24 \mathrm{~m}$.

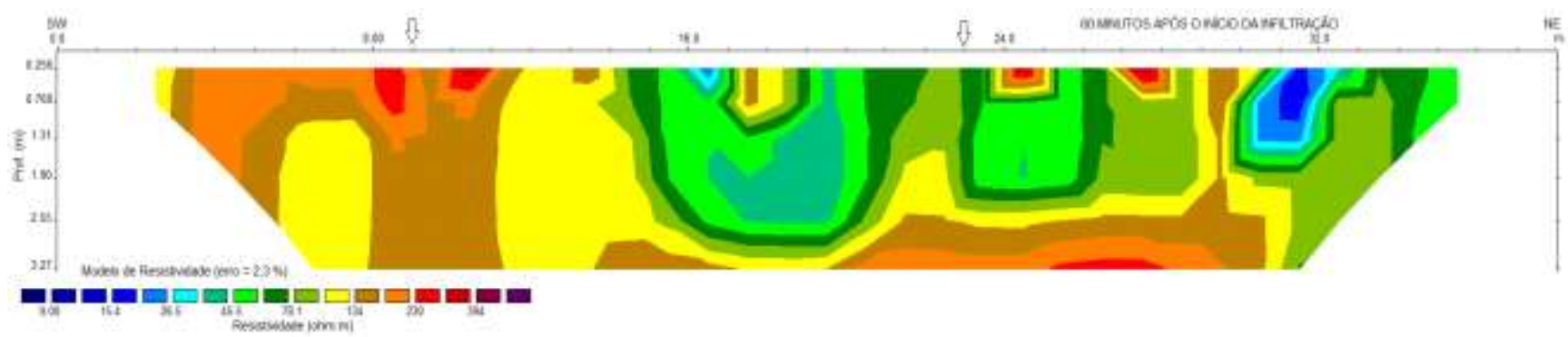

Figura 8 - Imagem obtida 60 minutos após o início da injeção. Os pontos de injeção em 9 m e 23 m são indicados pelas setas

Figure 8 - Image obtained 60 minutes after injection beginning. Injection at positions $9 \mathrm{~m}$ and $23 \mathrm{~m}$ are shown by the arrows

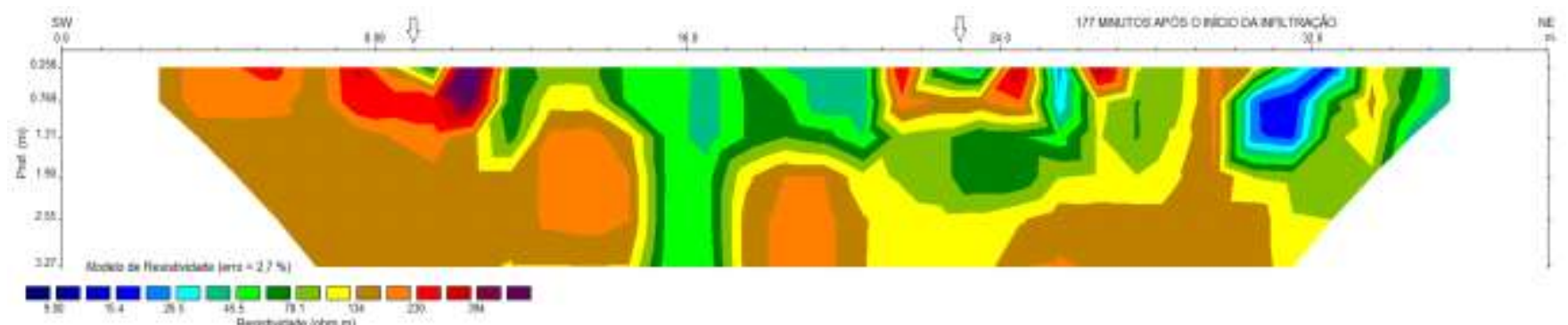

Figura 9 - Imagem obtida 177 minutos após o início da injeção. Os pontos de injeção em 9 m e 23 m são indicados pelas setas

Figure 9 - Image obtained 177 minutes after injection beginning. Injection at positions $9 \mathrm{~m}$ and $23 \mathrm{~m}$ are shown by the arrows 


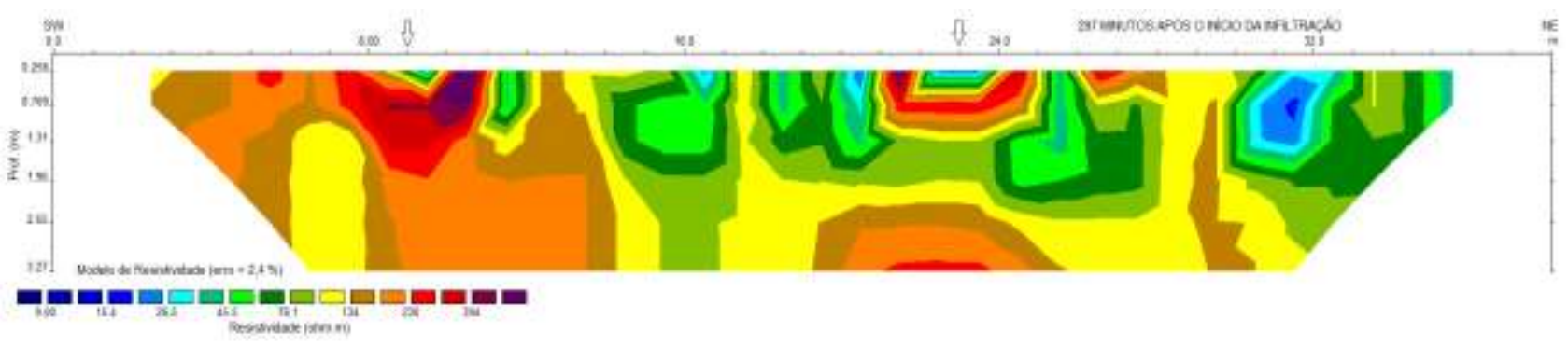

Figura 10 - Imagem obtida 297 minutos após o início da injeção. Os pontos de injeção em 9 m e 23 m são indicados pelas setas

Figure 10 - Image obtained 277 minutes after injection beginning. Injection at positions $9 \mathrm{~m}$ and $23 \mathrm{~m}$ are shown by the arrows

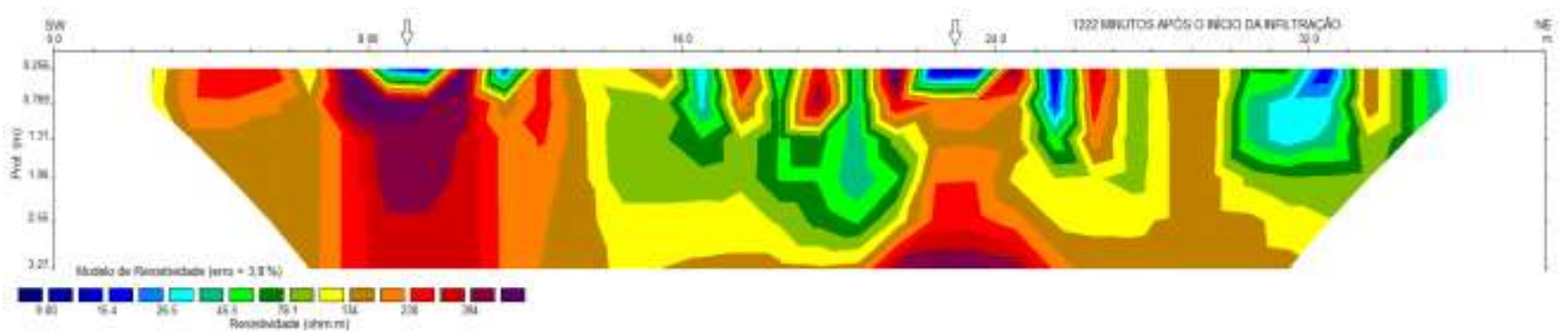

Figura 11 - Imagem obtida 1222 minutos após o início da injeção. Os pontos de injeção em 9 m e 23 m são indicados pelas setas

Figure 11 - Image obtained 1222 minutes after injection beginning. Injection at positions $9 \mathrm{~m}$ and $23 \mathrm{~m}$ are shown by the arrows

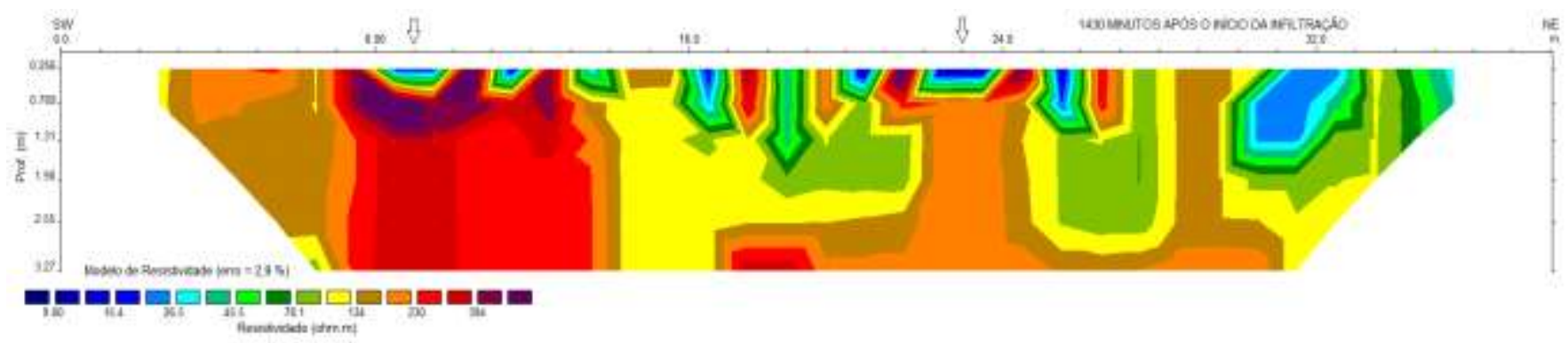

Figura 12 - Imagem obtida 1430 minutos após o início da injeção. Os pontos de injeção em 9 m e 23 m são indicados pelas setas

Figure 12 - Image obtained 1430 minutes after injection beginning. Injection at positions $9 \mathrm{~m}$ and $23 \mathrm{~m}$ are shown by the arrows

A partir de imagens obtidas em tempos diferentes, foi feita uma estimativa da velocidade com que a zona condutora causada pela solução salina se movimentou na subsuperfície, a fim de obter-se a velocidade do fluxo subterrâneo na zona não saturada. Para isso, foram medidas, nas imagens elétricas dos modelos, as distâncias entre o ponto de injeção e posições de avanço da zona condutora (rebaixamento da resistividade) ao longo do tempo, usando a escala de profundidade fornecida pelos modelos. A variação temporal foi obtida através da diferença entre o início da injeção e o tempo necessário para atingir determinada posição. $\mathrm{O}$ valor médio da velocidade de movimentação da zona condutora foi então calculado dividindo a distância pelo tempo. O procedimento foi realizado nas imagens obtidas durante a infiltração e naquelas em que ainda era observada a movimentação da pluma condutiva, mesmo após a infiltração ter sido encerrada. Esse fato foi observado até a imagem obtida 1430 minutos após o início da injeção, correspondendo a 823 minutos após o encerramento da injeção. A partir desse tempo, ocorreu um retorno às condições normais da subsuperfície.

A velocidade média às proximidades das posições 8 e $10 \mathrm{~m}$ foi estimada em $1,02 \times 10^{-3} \mathrm{~cm} / \mathrm{s}$ e próximo às posições 22 e $24 \mathrm{~m}$ em $1,32 \times 10^{-3}$ $\mathrm{cm} / \mathrm{s}$.

Segundo Araújo (2001) o valor da porosidade média da zona não saturada, para sedimentos 
similares aos que ocorrem na área de estudo, é igual a 0,19. Por sua vez, Rodrigues (1999), por meio de ensaios de permeabilidade realizados na área, estimou em $1,83 \times 10^{-4} \mathrm{~cm} / \mathrm{s}$ o valor da condutividade hidráulica média na zona não saturada. Essa média foi obtida a partir de ensaios de rebaixamento realizados em três poços rasos que produziram os valores $1,44 \times 10^{-4} \mathrm{~cm} / \mathrm{s}, 1,54 \times 10^{-4} \mathrm{~cm} / \mathrm{s}$ e $2,52 \times 10^{-4} \mathrm{~cm} / \mathrm{s}$. Dividindo-se a condutividade hidráulica média pela porosidade obtém-se uma estimativa de $0,96 \times 10^{-3} \mathrm{~cm} / \mathrm{s}$ para o valor da velocidade média do fluxo subterrâneo na área.

Existe uma pequena discrepância entre as estimativas de velocidade obtidas a partir do imageamento e a obtida a partir dos dados de porosidade de Araújo (2001) e de condutividade hidráulica de Rodrigues (1999), que pode ser devida ao valor obtido a partir de ensaios de permeabilidade ter levado em conta um fluxo essencialmente vertical (RODRIGUES, 1999), diferente do observado no imageamento elétrico.

Além disso, os valores de porosidade usados no cálculo são também aproximados, pois não foram determinados para os sedimentos da área estudada. Pode-se, portanto, concluir que a estimativa de velocidade do fluxo subterrâneo obtida através de imageamento, produziu resultados satisfatórios neste estudo.

\section{CONCLUSÕES}

A partir dos modelos obtidos por imageamento elétrico, estimou-se a velocidade média local do fluxo na subsuperfície em $1,02 \times 10^{-3} \mathrm{~cm} / \mathrm{s}$ e $1,32 \times 10^{-3} \mathrm{~cm} / \mathrm{s}$ nas proximidades dos dois pontos de injeção. Também foi possível observar,

\section{REFERÊNCIAS}

AGRAMAKOVA, Y. 2011. Time-lapse electrical resistivity tomography applied to cave sustainability (Barbados) and groundwater exploration (Saint Lucia). Cambridge, Massachusetts, 2011, 69 p. Tese (Mestrado em Geofísica). Massachusetts Institute of Technology, Department of Earth, Atmospheric, and Planetary Sciences.

ARAÚJO, P.P. Variações sazonais dos componentes nitrogenados, em aquífero livre na zona urbana de Santa Izabel do Pará, nordeste do estado do Pará. Belém, Pará, 2001, 113 p. Dissertação (Mestrado em Geologia). Universidade Federal do Pará, Centro de Geociências, Curso de Pós-Graduação em Geologia e Geoquímica.

BAESSA, M.P.M.; OLIVA, A.; KIANG, C.H. Imagea- através do movimento da pluma condutiva, um sentido do fluxo subterrâneo local que se espalha a partir das posições de injeção. Além disso, observou-se que esse movimento foi mais visível nas proximidades do ponto de injeção da posição $23 \mathrm{~m}$ do que no da posição $9 \mathrm{~m}$, sugerindo uma maior permeabilidade do material depositado nas proximidades de $23 \mathrm{~m}$.

A diferença na velocidade de fluxo estimada nas proximidades dos pontos de infiltração indica que o fluxo não é uniforme conforme é sugerido quando estimado através da metodologia hidrogeológica que utiliza observação em poços.

A discrepância entre as estimativas de velocidade obtidas a partir do imageamento e dos ensaios de permeabilidade pode ser devido aos ensaios terem levado em conta um fluxo essencialmente vertical. Além disso, a porosidade empregada para estimativa de velocidade a partir dos ensaios é aproximada, pois não foi obtida nos sedimentos da área de estudo.

\section{AGRADECIMENTOS}

Os autores agradecem à ALBRAS por ter cedido a área para a realização das medidas e ter fornecido parte do apoio logístico. Os autores também são gratos aos revisores da Revista Águas Subterrâneas, que, com suas sugestões, ajudaram a melhorar o texto do artigo. Um dos autores (AVBN) agradece à CAPES pela concessão de Bolsa de Estudos e o outro autor (JGL) ao Programa PARD da Universidade Federal do Pará, que financiou o levantamento de campo.

mento elétrico 3D em área contaminada por hidrocarboneto no polo industrial de Cubatão - SP. Revista Brasileira de Geofísica, v. 28, n. 4, p. 609-617, 2010.

BAHIA, V.E.; LUIZ, J.G.; LEAL, L.R.B.; FENZL, N.; MORALES, G.P. Diagnóstico sobre contaminação das águas subterrâneas na área do Parque Estadual do Utinga, Belém-PA, pelos métodos elétrico e eletromagnético. Revista Brasileira de Geofísica, v. 29, n. 4, p. 753$770,2011$.

BARKER, R.D.; MOORE, J. The appplication of timelapse electrical tomography in groundwater studies. The Leading Edge, v. 17, n.10, p. 1454-1458, 1998.

BENKABBOUR, B.; TOTO, E.A.; KAKIR, Y. Using DC resistivity method to characterize the geometry and 
the salinity of the Plioquaternary consolidated coastal aquifer of the Mamora plain, Morocco. Environmental Geology, v. 45, n. 4, p. 518-526, 2004.

BENSON, A.K.; PAYNE, K.L.; STUBBEN, M.A. Mapping groundwater contamination using dc resistivity and VLF geophysical methods-a case study. Geophysics, v. 62, n. 1, p. 80-86, 1997.

CASSIANI, G.; BRUNO, V.; VILLA, A.; FUSI, N.; BINLEY, A.M. A saline trace test monitored via timelapse surface electrical resistivity tomography. Journal of Applied Geophysics, v. 59, n. 3, p. 244-259, 2006.

COSTA, A.F.U.; DIAS, N.L.; ZANINI, L.F.; CORREA, O. Metodologia geofísica para detecção de contaminação de águas subterrâneas: caso do lixão da Estrada da Palha. A Água em Revista - Revista Técnica e Informativa da CPRM, v. 3, n. 6, p. 24-37, 1995.

CUNHA, L.F.J.; SHIRAIWA, S. Aplicação do método eletromagnético indutivo na investigação da pluma de contaminação da água subterrânea por resíduos de cromo de curtume. Revista Brasileira de Geofísica, v. 29, n. 1, p. 127-134, 2011.

DE FRANCO, R.; BIELLA, G.; TOSI, L.; TEATINI, P.; LOZEJ, A.; CHIOZZOTTO, B.; GIADA, M.; RIZZETTO, F.; CLAUDE, C.; MAYER, A.; BASSAN, V.; GASPARETTO-STORI, G. Monitoring the saltwater intrusion by time lapse electrical resistivity tomography: The Chioggia test site (Venice Lagoon, Italy). Journal of Applied Geophysics, v. 69, n. 3-4, p. 117 130, 2009.

DOETSCH, J.; LINDE, N.; VOGT, T.; BINLEY, A.; GREEN, A.G. Imaging and quantifying salt-tracer transport in a riparian groundwater system by means of 3D ERT monitoring. Geophysics, v. 77, n.5, p. B207B218, 2012.

FAROOQ, M; PARK, S.; KIM, J.H.; SONG, Y.S.; SABIR, M.A.; UMAR, M.; TARIQ, M.; MUHAMMAD, S. Time-lapse electrical resistivity investigations for imaging the grouting injection in shallow subsurface cavities. The Scientific World Journal, v. 2014, Article ID 178203, 7 p., 2014.

(http://dx.doi.org/10.1155/2014/178203).

FEITOSA, F.A.C.; MANOEL FILHO, J.; FEITOSA, E.; DEMÉTRIO, J.G.A. (Org.). Hidrogeologia: conceitos e aplicações - 3. ed. Rio de Janeiro: CPRM, 812 p., 2008.

FETTER, C. W. Applied hydrogeology. 4th Edition. New Jersey: Prentice Hall, 598 p., 2001.

FRENCH, H.; BINLEY, A. Snowmelt infiltration: monitoring temporal and spatial variability using time-lapse electrical resistivity. Journal of Hydrology, v. 297, n. 1-4, p. 174-186, 2004.

GRIFFITHS, D.H. Application of electrical resistivity measurements for the determination of porosity and permeability in sand-stones. Geoexplotration, v. 14, n. 3-4, p. 207-213, 1976.

HAGREY, S.A.; MÜLLER, C. GPR study of pore water content and salinity in sand. Geophysical Prospecting, v. 48, n. 1, p. 63-85, 2000.

HARARI, Z. Ground-penetrating radar (GPR) for imaging stratigraphic features and groundwater in sand dunes. Journal of Applied Geophysics, v. 36, n. 1, p. 43-52, 1996.

IPT (Instituto de Pesquisa Tecnológica do Estado de São Paulo). Estudo hidrogeológico para a definição da possibilidade de captação de água subterrânea na Usina de Alumínio da ALBRAS em Barcarena - PA. Relatório n 21-381, 1984.

KELLY, W.E. Geoelectric sounding for estimating aquifer hydraulic conductivity. Ground Water, v. 15, n. 6, p. 420-425, 1977.

LIMA, O.A.L. Estruturas geoelétrica e hidroquímica do sistema aquífero cristalino da bacia do alto rio Curaçá, semi-árido da Bahia. Revista Brasileira de Geofísica, v. 28, n. 3, p. 445-461, 2010.

LIMA, R.J.S. Levantamento geofísico para prospecção de água subterrânea no município de IrituiaPará. Belém, Pará, 1990, 36 p. Trabalho de Conclusão de Curso (Graduação em Geologia). Universidade Federal do Pará, Centro de Geociências.

LIMA, O.A.L.; NIWAS, S. Estimation of hydraulic parameters of shaly sandstone aquifers from geoelectrical measurements. Journal of Hydrology, v. 235, n. 12, p. 12-26, 2000.

LIMA, W. N.; KOBAYASHI, C. N. Sobre o quimismo predominante nas águas do sistema flúvio-estuarino de Barcarena, Pa. Geochimica Brasiliensis, v. 2, n.1, p.5371, 1988.

LU, Q.; SATO, M. Estimation of hydraulic property of an unconfined aquifer by GPR. Sensing and Imaging, v. 8, n. 2, p. 83-99, 2007.

MEDEIROS, W.E.; LIMA, O.A.L. A geoelectrical investigation for ground water in crystalline terrains of Central Bahia, Brazil, Ground Water, v. 28, n. 4, p. 518-523, 1990.

MENDES, M.F.; ALVES, J.G.V.; SILVA, M.W.C. Prospecção geofísica para identificação de zonas aquífe- 
ras na formação Codó em Jacundá/PA. Revista Águas Subterrâneas, v. 28, n. 1, p. 62-79, 2014.

NIWAS, S.; LIMA, O.A.L. Aquifer parameter estimation from surface resistivity data. Ground Water, v. 41, n. 1, p. 94-99, 2003.

NUNES, L.P.M.; LUIZ, J.G. Caracterização geoelétrica de área de curtume localizada no Distrito Industrial de Icoaraci, Belém-Pará. Revista Brasileira de Geofísica, v. 24, n. 4, p. 467-481, 2006.

OLIVEIRA, M.A.S. Aplicabilidade do método geofísico de eletrorresistividade na pesquisa de água subterrânea em rochas cristalinas na região de Conceição do Coité-BA. Salvador, Bahia, 2011, 67 p. Monografia de final de curso (Graduação em Geologia), Universidade Federal da Bahia, Instituto de Geociências.

ORELLANA, E. Prospeccion geoelectrica en corriente continua (2 ed.). Paraninfo, Madrid, 578 p., 1982.

PONZINI, G.; OSTROMAN, A.; MOLINARI, M. Empirical relation between electrical transverse resistance and hydraulic transmissivity. Geoexploration, v. 22, n. 1, p. 1-15, 1984.

RODRIGUES, S.F.S. Vulnerabilidade do aquífero livre na área de lançamento de rejeitos sólidos da ALBRAS e avaliação preliminar dos seus riscos de contaminação. Belém, Pará, 1999, 66 p. Trabalho de Conclusão de Curso (Graduação em Geologia). Universidade Federal do Pará, Centro de Geociências.

SAUCK, W.A.; ATEKWANA, E.A.; NASH, M.S. High conductivities associated with an LNAPL plume imaged by integrated geophysical techniques. Journal of Environmental and Engineering Geophysics, v. 2, n. 3, p. 203-212, 1998.

SOUPIOS, P.M.; KOULI, M.; VALLIANATOS, F.; VAFIDIS, A.; STAVROULAKIS, G. Estimation of aquifer hydraulic parameters from surficial geophysical methods: a case study of Keritis Basin in Chania (Crete - Greece). Journal of Hydrology, v. 338, n. 1, p. 122$131,2007$.

SOUSA, G.B.; LUIZ, J.G. Groundwater prospection in the municipality of Piçarra-PA using very low frequency and resistivity. Revista Brasileira de Geofísica, v. 30, n. 3, p. 361-372, 2012.

SOUZA FILHO, O.A.; OLIVEIRA, R.G.; RIBEIRO, J.A.; VERÍSSIMO, L.S.; SÁ, J.U. Interpretação e modelagens de dados de eletrorresistividade para locações de poços tubulares no aquífero fissural da área-piloto Juá, Irauçuba - Ceará. Revista de Geologia, v. 19, n. 1, p. 721, 2006.

TANCREDI, A.C.F.N.S. Recursos hídricos subterrâneos de Santarém: fundamentos para uso e proteção. Belém, Pará, 1996, 153 p. Tese (Doutoramento em Geoquímica). Universidade Federal do Pará, Centro de Geociências, Curso de Pós-Graduação em Geologia e Geoquímica.

WILKINSON, P.B.; MELDRUM, P.I.; KURAS, O.; CHAMBERS, J.E.; HOLYOAKE, S.J.; OGILVY, R.D. High-resolution Electrical Resistivity Tomography monitoring of a tracer test in a confined aquifer. Journal of Applied Geophysics, v. 70, n.4, p. 268-276, 2010 . 